Apidologie, 1984, 15 (2), 91-98

\title{
DIE IMKERORGANISATION UND PROFESSOR DR. DR. FRIEDRICH RUTTNER
}

\author{
Dr. F. GNADINGER \\ D - 7768 Stockach (B.R.D.)
}

Wer das Glück hatte, ein Stück des Lebensweges mit Professor Dr. Dr. F. RutTNer gehen zu dürfen, wird große Achtung und Bewunderung für ihn mitgenommen haben.

Die folgenden Zeilen sind sehr persönlich und sollen an Hand von wenigen Beispielen über die Zusammenarbeit des Jubilars Prof. Dr. RuTtner mit den Imkerorganisationen, insbesonders dem Deutschen Imkerbund, erzählen.

Ich begegnete Herrn Dr. Friedrich RutTNER zusammen mit seinem Brudei Hans zum ersten Mal im Jahre 1962. Bis dahin hatte ich viel über die Tätigkeiten der Brüder Friedrich und Hans gehört und einiges gelesen - eigentlich waren die beiden für mich bereits bekannt und berühmt.

\section{PAARUNGSBIOLOGIE UND ZUCHT}

1962 wurde mir die Leitung des südbadischen Imkerverbandes anvertraut.

Im Rahmen der Aktivierung der Fachgebiete bestand die Notwendigkeit, ein Konzept für die züchterischen Zielsetzungen im Verband zu erstellen. Schließlich machte ich den Vorschlag, eine Reise zu den Ruttners nach Lunz am See zu macnen. Die kleine Gruppe badischer Imker (P. Weissharr, A. Walterer und ich) gehörte damit zu den vielen Ratsuchenden, die zur Bienenstation nach Lunz pilgerten.

Wir sahen die Erfolge in der Zucht der Carnica-Biene, den vorbildlichen Leistungsprüfstand und noch vieles andere mehr. Nach langen Gesprächen mit den Brüdern RUTTNER waren wir davon überzeugt, daß die Zuchtarbeit im badischen Verband nach diesem Vorbild aufgebaut werden sollte. 
Es war für die Ruttners selbstverständlich, mit Vertreten einer Imkerorganisation oder mit Praktikern in geduldigem Für und Wider die Probleme zu diskutieren. Sicher waren fachliche Überlegungen nicht der ausschließliche Grund; es war auch die Freude, mit anderen Menschen sprechen zu können und die $\mathrm{Be}$ friedigung, den Imkern in einer Zeit manchmal verworrener Meinungsbildung helfen zu dürfen.

Als wir erfuhren, daß für den Aufbau der Abteilung für Bienengenetik an der Biologischen Station Lunz nach dem Krieg der Österreichische Imkerbund zehn Jahre lang einen Teil der notwendigen Kosten aufbrachte, spürten wir Bewunderung für die Aufgeschlossenheit und Solidarität der für die Bienenzucht Verantwortlichen in Österreich. Der Leistungsprüfstand konnte damals auf einen 10jährigen Bestand zurückblicken; er wurde zu einem Pilot-Projekt im europäischen Raum.

1963 begegneten wir uns wieder auf dem APIMONDIA-Kongreß in Prag, wo die Erkenntnisse über Mehrfachpaarung der Bienenköniginnen, Drohnensammelplätze und Versuche über künstliche Samenübertragung besonders lebhaft diskutiert wurden. Dort wurde Dr. Friedrich RutTner zum Präsidenten der Biologie-Kommission der APIMONDIA gewählt, ein Amt, das er bis heute inne hat.

Es war eine Zeit fundamentaler Entdeckungen, die von den Imkern begierig aufgenommen wurden. Ein wesentlicher Punkt war die Frage der Sicherheit der Belegstellen. Man suchte nach Anpassungen an die neuen Erkenntnisse. Diese konnten nur gemeinsam von den Fachwissenschaftlern mit den Imkerorganisationen gefunden werden. Eine Zusammenarbeit war vorprogrammiert.

Die Zucht bei großen Haustieren liegt bei staatlichen Behörden und halbstaatlichen Verbänden, dort gelten Gesetze und Verordnungen. Für die Zucht der Bienen müssen verbandseigene «Zuchtordnungen oder Zuchtrichtlinien 》 geschaffen und befolgt werden. Der Beitrag der Wissenschaftler liegt in den Erkenntnissen aus vielen Versuchen in der Paarungsbiologie und Genetik. Der Beitrag der Imkerorganisationen liegt in der praktischen Durchführung unter Beachtung der Grenzen des Möglichen. Es entstand eine fruchtbare Zusammenarbeit zwischen dem Lunzer Institut und den Imkerverbänden Deutschlands und Österreichs, wobei in Deutschland Dr. K. DreHer als weiterer Experte mitwirkte.

Wenn Hergebrachtes durch neue Erkenntnisse überholt wird, ist die Zeit reif für eine Bestandsaufnahme auf breiter Grundlage; auch müssen die Weichen für die Zukunft gestellt werden. Der inzwischen zum Professor avancierte Dr. Friedrich RutTNer und Hans RutTner iniziierten das Symposium der APIMONDIA uiber Probleme der Paarungskontrolle und Selektion bei der Honigbiene 1972 in Lunz. Es war eine der schönsten Tagungen, die ich je besucht habe. Eine Begegnung, die fachlich und organisatorisch von hohem internationalen Niveau war. 
Eine neue Zeit war eingeleitet : die gezielte Paarung über künstliche Besamung der Bienenköniginnen eroberte die Imkerwelt. Noch war es in Europa eine Sache der Wissenschaftler, aber es sollte sich bald ändern.

Am 1. November 1964 übernahm Dr. F. RutTner als Nachfolger von Dr. A. Gontarski das Institut der Polytechnischen Gesellschaft Frankfurt in Oberursel. Er kam damit noch intensiver mit deutschen Imkern und deren Organisationen in Berührung. Als Präsident des DIB hatte ich die Freude, viele Jahre mit dem Jubilar in ungetrübtem Vertrauen zusammenarbeiten zu dürfen.

Prof. Dr. Rutrner trat 1969 an den DIB mit der Mitteilung heran, daß die Methodik der künstlichen Besamung von Bienenköniginnen ausgereift und somit die Zeit gekommen sei, ihre züchterischen Vorteile für die Praxis zu nutzen. Er machte den Vorschlag, eine Besamungstation in Oberursel zu errichten. Wirtschaftlicher Träger sollte der DIB sein, für die fachliche Beratung stellte er sich und sein Institut zur Verfügung. Prof. Dr. Ruttner stellte die Idee in einem Vortrag bei einer Tagung der Arbeitsgemeinschaft Deutscher Tierzüchter in München vor. Künstliche Besamung bei Bienenköniginnen war für die Großtierzüchter eine fremde und wunderliche Sache. Prof. Dr. F. RuTriner gelang es, seinen Vortrag so spannend zu machen, daß «mäuschenstill » zugehört wurde. Die Station wurde geschaffen, ein Zuschuß des Bundeslandwirtschaftsministeriums erleichterte den Start.

Die Imker verstanden sehr schnell die Bedeutung dieser Station. Die besamten Königinnen wurden gerne gekauft, so daß dic Kapazität bald nicht mehr ausreichte. Mitarbeiter wurden nach Bir Bou Rebka in Tunesien geschickt, wo zu früherer Jahreszeit besamt werden konnte als in Mitteleuropa. Dic Königinnen wurden anschließend in die BRD versandt.

Nach Einrichtung der Bienenabteilung an der Hessischen Landesanstalt für Leistungsprüfungen in der Tierzucht wurde die Station auf Wunsch des Landesverbandes Hessischer Imker nach Kirchhain verlegt. Dr. V. Maul führte das Werk weiter. Die kommerzielle künstliche Besamung von Bienenköniginnen ist heute Routine.

Es ist ein schönes Beispiel für die gelungene gemeinsame Pionierarbeit auf einem heute selbstverständlichen Gebiet, wobei die Vcrdienste von Prof. Dr. F. RUTTNER hervorgehoben werden müssen.

\section{VARROATOSE}

Auf dem Internationalen Bienenzüchterkongreß der APIMONDIA in Maryland 1967 hörte ich ein kurzes Referat von Prof. Dr. PolTEv (UdSSR) über das 
Auftreten einer gefährlichen Milbenkrankheit bei den Bienen in Primorje/Sibirien. Er sprach von großen Verlusten unter den Bienenvölkern. Niemand ahnte damals, welche Bedeutung diese Krankheit, verursacht von der Milbe Varroa jacobsoni, einmal gewinnen würde.

In den Jahren danach war es ruhig bis etwa 10 Jahre später durchsickerte, daß diese Milbe in der UdSSR erhebliche Verluste unter den Bienenvölkern verursachte.

Durch Einfuhr von Bienenköniginnen aus der UdSSR 1964-1965 wurde die Milbe nach Bulgarien eingeschleppt. Von da aus verbreitete sie sich in die angrenzenden Länder.

Veröffentlichungen über die neue Seuche waren in der deutschen Literatur mager. Wir hörten zwar von Wissenschaftlern aus den Oststaaten, insbesonderes der Sowjetunion darüber, aber im Grunde nahm sie niemand recht ernst.

Am Rande des Symposiums über Bienengenetik in Moskou 1977 suchte ich ein Gespräch mit Prof. Poltev. Er erzählte vom Auftreten, den Verlusten und der Bekämpfung in der UdSSR und brachte einige tote Milben mit. Es gab danach eine Reihe von Besprechungen und Tagungen : 1976 in Sofia unter Wissenschaftlern der Oststaaten, wovon wir im Westen zunächst nichts erfuhren; 1978 in Bukarest ein Seminar über Prophylaxe und Therapie der Varroatose, getragen von der APIMONDIA und dem Internationalen Tierseuchenamt.

Anfang 1977 wurden bei einigen Instituts-Völkern in Oberursel VarroaMilben gefunden. Höchster Alarm !

Prof. Dr. F. RutTNer stellte seine bisherigen Forschungen über Bienenbiologie, Paarung und Genetik um und konzentrierte sich auf die Ausmerzung des Varroa-Befallsherdes.

Die in den Oststaaten bis dahin verwendeten Bekämpfungsmittel hatten nicht vermocht, Herde auszurotten. Man erfuhr von einem guten Medikament in Japan : Varrostan-Bayer/Japan. Es sollte wirksamer sein als bisher bekannte Medikamente.

Da die Zeit drängte, wandte sich Prof. Dr. F. Rutrner an den DIB. Über die Arbeitsgemeinschaft der Institute für Bienenforschung stellte man für die Beschaffung von Varrostan und weiteren Notwendigkeiten DM 100 000,00 zur Verfügung. Es war eine Nothilfe, deren Voraussetzung ein Vertrauensverhältnis zu Prof. Dr. F. Ruttner und in sein wissenschaftliches Können war.

Leider brachte die Anwendung von Varrostan nicht den gewünschten Erfolg. Zahlreiche weitere Mittel wurden seitdem in Oberursel geprüft. Das ideale Medikament zur Sanierung der Bienenvölker ist bis heute nicht gefunden worden. 
Mit dem systemisch wirksamen K-79 (Chlordimeformhydrochlorid) war man aber auf einem guten Weg. Leider konnte die Zulassung des Bundesgesundheitsamtes wegen bestimmter Nebenwirkungen nicht erreicht werden.

Prof. Dr. F. RutTner schlug vor, ein Symposium der APIMONDIA durchzuführen : Varroatose und deren Bekämpfung, um Rückblick und Ausschau zu halten. Das Symposium fand - von der Biologie- und Pathologickommission der APIMONDIA gemeinsam getragen - im Herbst 1980 in Oberursel/Bad Homburg statt. Der DIB und Landesverband Hessischer Imker unterstützen das Vorhaben nach Kräften.

Eine sehr gute Tagung, von der ein Strom neuer Erkenntnisse hinausging und viele Neuigkeiten zu uns kamen.

Nach vielen Testversuchen kam Folbex-VA-Neu (Brompropylat) der Firma CIBA-GEIGY auf den Markt. Es ist im Februar 1982 vom Bundesgesundheitsamt als Tierarzneimittel gegen die Varroatose zugelassen worden. Für die BRD stand damit wenigstens ein Medikament zur Verfügung.

Prof. Dr. RutTner arbeitete auch nach seiner Verabschiedung in den Ruhestand am 31. Mai 1979 im Institut weiter. Sein Nachfolger Professor Dr. N. Koeniger trat in seine Fußstapfen.

Es wird außerordentlich bedauert, daß die Varroatose in der BRD bei Bienenvölkern im Oberurseler Institut zuerst aufgetreten ist; sicherlich wäre sie wenige Jahre später auch gekommen. Neben allem Belastendem muß man die menschliche Größe von Prof. Dr. F. Ruttner sehen : keine Resignation, sondern Aufnahme des Kampfes gegen die Varroatose mit größter Energie.

Ein Besuch aus Nizza, Dr. M. E. Colın, hatte die Idee, Forschungen über Biologie und Bekämpfung der Varroatose auf europäischer Ebene durchzuführen. Inzwischen wurden nämlich auch Herde in Italien, Frankreich, Finnland u.a. festgestellt. Bekämpfung allein mit Milben-tötenden Medikamenten konnte nicht «das Beste sein» - ein Unbehagen wegen der ständigen Anwendung von Medikamenten machte sich breit.

So entstand die Idee des Europäischen Forschungsprogrammes. Im Einvernehmen mit dem Institut in Oberursel stellte der Deutsche Imkerbund den Antrag an die Kommission in Brüssel. Er wurde von der COPA unterstützt.

Hauptziel waren Forschungen zur Bekämpfung der Varroatose mit biotechnischen Verfahren in weitestem Sinne.

Um Wissenschaftler aus verwandten Disziplinen und Verwaltungsbeamte der EG mit der besonderen Problematik vertraut zu machen, wurde im Februar 1983 ein Symposium in Wageningen/Holland organisiert. 
Die Finanzierung des Forschungsprogrammes ist inzwischen trotz schlechter Finanzlage der EG gesichert, wenn auch nicht im gewünschten Ausmaß. Aber man kann beginnen - ein gemeinsamer Erfolg der Professoren Dr. F. RutTNer, Dr. N. Koeniger und Dr. M. E. Colin u.a. sowie der Imkerorganisationen!

\section{VORTRÄGE}

Wenn man die Bienenzeitschriften der 60iger bis 80iger Jahre durchsieht, findet man zahlreiche Veröffentlichungen und Vorträge von Prof. Dr. F. RutTnER. Wo immer es möglich war, entsprach er den Bitten von Imkerverbänden und -vereinen, Vorträge zu halten. Er verstand es meisterhaft, wissenschaftliche Probleme so darzustellen, daß sie ein einfacher Imker verstehen konnte.

Er hatte auch keine Scheu, kritische Themen anzupacken. Ich erinnere an einen Vortrag in Mannheim 1975, wo er sich mit der aktuellen Hybrid-Kreuzungszucht auseinandersetzte oder an Vorträge über die Varroatose, in denen er auf vorwurfsvolle Fragen der Imker antwortete.

Seine Vorträge waren stets aktuell und fanden großen Zulauf. Das Repertoire bewegte sich über viele Bereiche, nicht nur die erwähnten. Er machte sich z.B. Gedanken über Bienen und Umwelt im biologischen Vergleich zu anderen warmund kaltblütigen Lebewesen (Internationales Bodensee-Imkertreffen 1982 in Bregenz).

Gerade dieser Vortrag zeugt von seiner Ehrfucht vor der Natur. Es wurde von einer «Partnerschaft mit den Mitgeschöpfen, wie sie einst Franz von Assisi geprägt hat » gesprochen, «die ein richtiger Imker heute seiner Mitwelt vorzeigen kann》 und von einer «Partnerschaft IMKER-BIENE, wie sie täglich praktiziert wird als Modell einer möglichen Zukunftsentwicklung : Nutzen durch den Menschen, aber Respektierung der lebenden Kreatur in ihrer Eigenständigkeit mitsamt der von ihr bewohnten Landschaft ».

Das Symposium Bienenmuseum und Geschichte der Bienenzucht im August 1977 in Freiburg i.Br. und der APIMONDIA-Kongreß in Athen 1979 veranlaßten ihn, sich mit der Geschichte der Bienenzucht zu befassen. Er hielt mehrere Vorträge und verfaßte eine Broschüre : Historische Entwicklung des Bienenkorbes.

Der großen Bedeutung halber möchte ich auch auf die wissenschaftliche Zeitschrift Apidologie hinweisen. Sie ist ein Gemeinschaftswerk der französischen I.N R A. und des Deutschen Imkerbundes, welches auf Initiative unseres hochverehrten Dr. J. Louveaux zustande gekommen ist. 
Nachdem Prof. Dr. O. WaHL, der erste deutsche Schriftleiter, nach vielen Jahren altershalber ausschied, trat Prof. Dr. F. RutTner an seine Stelle.

Sicherlich sind die wirtschaftlichen Probleme wegen der relativ geringen Abonnentenzahl dieser fachwissenschaftlichen Zeitschrift schwierig. Trotzdem besteht bei allen Beteiligten der ungebrochene Wille zur Zusammenarbeit und zum Erhalt dessen, was im gemeinsamen Interesse aufgebaut wurde.

\title{
EHRUNGEN
}

Der Deutsche Imkerbund ließ Prof. Dr. F. Ruttner als Anerkennung für seine großartigen Leistungen und als Ausdruck des Dankes für seine vielen Arbeiten zugunsten der Imkerei und für seine Zusammenarbeit Ehrungen zukommen :

Zeidlermännchen anläßlich seines 60. Geburtstages 1974 ;

Ehrenimkermeister anläßlich seines Abganges aus dem Institut in Oberursel am 29. September 1980.

Wir wissen, daß er von der Regierung seines Heimatlandes Österreich und anderen Ländern hohe staatliche Auszeichnungen erhalten hat.

Die Imkerorganisationen gratulieren dem Jubilar zu seinem 70. Geburstag und wünschen volle Kraft und Gesundheit für viele Jahre!

\author{
RESUMÉ \\ L'ORGANISATION DES APICULTEURS ALLEMANDS \\ ET LE PROFESSEUR DR. FRIEDRICH RUTTNER
}

Friedrich RUTTNER et son frère Hans RUTrNER ont fortement marqué l'apiculture en Allemagne. A partir de leurs travaux scientifiques sur l'accouplement multiple et les lieux de rassemblement de mâles, ils ont tous deux contribué à l'amélioration de l'apiculture, restant constamment en liaison avec les apiculteurs et leurs organisations.

En 1964 F. Rutrner prit la direction de l'Institut für Bienenkunde (Polytechnische Gesellschaft) à Oberursel. C'est là que fut créée, en collaboration avec le Deutscher Imkerbund, la première station d'insémination artificielle. Cette station, située aujourd'hui à Kirchhain, fut le premier endroit où l'insémination artificielle a été utilisée avec succès sur une large échelle dans le travail pratique de sélection.

Après l'arrivée de la varroose en Allemagne fédérale en 1977, F. Rutrner a coordonné la campagne de lutte contre cette «nouvelle» maladie. En étroite collaboration avec les apiculteurs, leurs organisations et les services vétérinaires, il a testé les produits existants et, en raison de leurs effets indésirables, mit au point avec ses collaborateurs d'Oberursel de meilleurs médicaments 
pour traiter la varroose. Ses études sur la biologie de Varroa constituent une base de recherche pour mettre au point des traitements biologiques pour les colonies infectées.

Durant sa retraite F. RuTTNER poursuit son travail à Oberursel. Il a institué une étroite collaboration européenne dans la recherche sur Varroa, susceptible d'aboutir à un groupe de travail financé par la CEE. C'est le résultat d'une action commune entre les organisations apicoles européennes et les instituts de recherche.

F. Rutrner a consacré beaucoup d'efforts et de temps à des conférences et des articles pour les apiculteurs. Ses avis et son aide ont toujours été basés sur une recherche biologique approfondie et une large expérience personnelle dans le domaine de la biologie de l'abeille. Sa contribution à l'apiculture lui a valu de nombreux honneurs et décorations.

\section{SUMMARY}

\section{THE GERMAN BEEKEEPERS ASSOCIATION AND PROFESSOR DR. FRIEDRICH RUTTNER}

Honey bee breeding in the Federal Republic of Germany was significantly influenced and improved by Frjedrich RutTNer and his brother Hans RUTTNER. Based on their scientific work on multiple mating and drone congregation areas they were in a continuous discussion with beekeepers and their organisations, which led to a better design of mating stations (Belegstellen).

In 1964 F. RutTNer became director of the Bee Research Institute (Institut für Bienenkunde, Polytechnische Gesellschaft) in Oberursel. In close cooperation with German Beekeepers Association he started to introduce artificial insemination in the practical breeding work. The first station was founded where beekeepers brought their own qu.ens and drones and got them artificially mated. Later this station was moved to Kirchhain where it operates successfully up to the present time.

After the detection of Varroatosis in Germany in 1977 F. RUTTNER coordinated the campaign against this «new》 honey bee disease. Together with beekeepers, their organisations and the official veterinary service, he tested the existing medications and because of their unsatisfactory effects, he and his collaborators in Oberursel started to develop better drugs for the therapy of varroatosis. His research on the biology of Varroa laid the basis for the search for biological treatments of infested bee colonies.

After his retirement, F. RUTTNER continued his work in Oberursel. He initiated a close European cooperation in Varroa research which eventually led to the foundations of a European working group fonded by the Common Market. This success again was the result of a joint operation between the European beekeeping organisations and the research institutes.

F. RUTTNER devoted much effort and time to talks and presentations for beekeepers. His advice and assistance were always based on sound biological research and a broad personal experience in bee biology. He received many honours and decorations for his dedication to the benefit of beekeeping. 